Chapter 14

\title{
Infrared Surface Plasmon Spectroscopy of LiVing CELLS
}

\author{
M. Golosovsky, ${ }^{*}$ V. Yashunsky, A. Zilberstein, T. Marciano, \\ V. Lirtsman, D. Davidov, B. Aroeti \\ The Racah Insitute of Physics, The Hebrew \\ University of Jerusalem, Jerusalem 91904, Israel \\ Department of Cell and Animal Biology, \\ The Alexander Silberman Institute of Life Sciences, \\ The Hebrew University of Jerusalem, 91904 Jerusalem, Israel
}

\begin{abstract}
We report a spectroscopic technique that combines the Fourier-Transform Infrared Spectroscopy with the Surface Plasmon Resonance. This tool enables sensitive infrared spectroscopy of liquid and solid objects in the attenuated total reflectance mode. The FTIR-SPR technique is similar to FTIR-ATR technique but has higher sensitivity due to resonance amplification of the surface electric field. The label-free FTIR-SPR technique is especially advantageous for living cell studies since it combines spectroscopic information inherent to FTIR with structural information provided by the Surface Plasmon Resonance. We discuss the FTIR-SPR technique for label-free studies of cell sedimentation and spreading on substrate and for surface plasmon spectroscopy.
\end{abstract}

PACS 05.45-a, 52.35.Mw, 96.50.Fm.

Keywords: surface plasmon, infrared, FTIR, biosensor, cell culture

\section{Introduction}

Surface plasmon resonance (SPR) technique $[1,2,3,4]$ became an important research tool in biophysics. SPR measures refractive index and optical absorption with high sensitivity and is particularly advantageous for biosensing since it can monitor kinetics of biological processes on surfaces and in solutions in real time. Although most SPR applications focus

\footnotetext{
*E-mail address: golos@cc.huji.ac.il
} 
on the visible range, the SPR setups operating in the near-infrared have been reported as well $[5,6,7,8,9]$. The advantages of the near-infrared range are reduced conductor losses and concomitant increase in sensitivity. Refs. [5, 6] pioneered the surface plasmon excitation using FTIR spectrometer. This resulted in the powerful near-infrared surface plasmon technique with wavelength interrogation that offers several advantages over conventional SPR techniques based on angular interrogation. Recently, we extended the FTIR-SPR technique into mid-infrared range by using metallic mirrors and $\mathrm{ZnS}$ prism $[10,11]$.

The FTIR-SPR technique is particularly useful for real-time and quantitative measurements of dynamic processes in living cells. Indeed, the SPR technique in the visible range has been successfully used in cell culture studies $[14,15,16,17]$ whereas the main advantage of the SPR over conventional optical methods is that it is label-free. However, since in the visible range the penetration depth of the surface plasmon wave into the cell is only $\sim 0.1 \mu \mathrm{m}$, it is mostly sensitive to the shape/mass redistribution occurring in the vicinity of cell basal membrane. In other words, the surface plasmon in the visible range senses mostly cell adhesion and is less sensitive to the processes occurring deeper in the cell. Here, the infrared surface plasmon is more advantageous since its penetration depth of a few $\mu \mathrm{m}$ is comparable to the cell height and it can sense the intracellular processes as well.

In the context of biosensing the FTIR-SPR technique has several attractive features: high sensitivity to refractive index changes, variable probing depth, spectroscopic capabilities. So far we used this technique to study alterations in membrane cholesterol levels in HeLa cells [12], de novo formation of early endocytic transport vesicles in response to transferrin internalization by human melanoma cells [13], and detection of glucose uptake by erythrocytes [11].

While spectroscopic use of the SPR technique in the visible and near-infrared ranges has been demonstrated $[7,18,19,20]$, the most useful spectroscopic information appears usually in the mid-IR range. So far, FTIR spectroscopy has been performed in the transmission/reflection mode or using ATR setup. This is an important challenge to combine mid-IR spectroscopy and SPR. While conventional FTIR techniques aim at measuring absorption, the SPR technique measures a complementary parameter- refractive index.

The FTIR-SPR technique can be particularly useful in living cell cell studies. So far, FTIR absorption spectroscopy of living cells has been performed using synchrotron radiation [21, 22], ATR [23, 24, 25, 26], or evanescent field of the infrared fiber [27]; while the surface plasmon has been mostly used for detecting structural modifications in cells. Combination of surface plasmon resonance with spectroscopy can be particularly useful in the study of drug penetration into cells, since it can measure not only the spectroscopic signature of drug but the cell reaction to this drug as well.

\section{Methodology}

The surface plasmon resonance at the metal-dielectric interface is usually excited in the Kretschmann's geometry (Fig.2) that employs high-refractive index prism operating in the regime of attenuated total reflection (ATR). The prism is coated with a thin metal film and the analyte (liquid, thin film, or living cell layer) is in contact with it. The resonance appears 
when the surface plasmon wave vector satisfies the phase matching condition

$$
k_{s p}^{\prime}=k_{x}=k_{0} n_{p} \sin \Theta
$$

where $k_{0}$ is wave vector in free space, $n_{p}$ is the refractive index of the prism, $\Theta$ is the incident angle at the prism-metal interface. The real and imaginary parts of the surface plasmon wave vector are

$$
k_{s p}^{\prime}+i k_{s p}^{\prime \prime}=k_{0}\left(\frac{\epsilon_{m} \epsilon_{d}}{\epsilon_{m}+\epsilon_{d}}\right)^{1 / 2}
$$

where $\epsilon_{d}(\lambda)$ is the dielectric permittivity of the analyte and $\epsilon_{m}(\lambda)$ is the (negative) dielectric permittivity of the metal. For fixed incident angle and wavelength interrogation, the resonant condition reduces to:

$$
n_{d}^{2}=\epsilon_{d}=\frac{\left|\epsilon_{m}\right| n_{p}^{2} \sin ^{2} \Theta}{\left|\epsilon_{m}\right|+n_{p}^{2} \sin ^{2} \Theta}
$$

The surface plasmon resonance appears as a reflectivity minimum at certain wavelength $\lambda$ which is inexplicitly given by Eq. 3 . The reflectivity at the resonance can be usually approximated by a Lorentzian [28]

$$
R=\left|r_{F}\right|^{2} \frac{1-k_{s p}^{\prime \prime} \Gamma}{\left(k_{x}-k_{s p}^{\prime}\right)^{2}+\left(k_{s p}^{\prime \prime}+\Gamma\right)^{2}}
$$

where $r_{F}$ is the Fresnel reflection coefficient at the prism-metal interface and $\Gamma$ is the radiation (coupling) loss which appears when the surface plasmon propagates along a thin metal film rather than along a bulk metal surface. The minimal reflectivity

$$
R_{\min }=\left|r_{F} \frac{k_{s p}^{\prime \prime}-\Gamma}{k_{s p}^{\prime \prime}+\Gamma}\right|
$$

occurs at resonance condition, $k_{x} \approx k_{s p}^{\prime}$. For optimal coupling, which is achieved by proper choice of the metal film thickness, $\Gamma=k_{s p}^{\prime \prime}$ and $R_{\text {min }}=0$.

For inhomogeneous medium whose properties vary in the $z$-direction, the refractive index of the analyte sensed by the evanescent wave is

$$
n_{d}^{e f f}=\delta^{-1} \int_{0}^{\infty} n_{d}(z) e^{-\frac{z}{\delta}} d z
$$

where $\delta$ is the surface plasmon penetration depth,

$$
\delta_{z}=\frac{\lambda}{4 \pi n_{d}}\left(\left|\epsilon_{m}\right|-n_{d}^{2}\right)
$$

The surface plasmon in the infrared range is usually used for highly sensitive refractometry. Tiny variations of the analyte refractive index, $\Delta n$, are found from the shift of the surface plasmon resonance

$$
\Delta \nu=S \Delta n
$$


where $\nu$ is the resonance wave number and $S$ is the bulk sensitivity which can be found from Eqs.1,3.

In comparison to its visible range counterpart, the FTIR-SPR allows wavelength interrogation in addition to conventional angular interrogation. However, the requirements to conducting film are more stringent than for the surface plasmon technique in the visible range. On the one hand, the infrared surface plasmon is very narrow (and is therefore more sensitive) due to decreased conducting losses. On the other hand, to get full advantage of this enhanced sensitivity, the conducting film should be very thin.

In the context of living cells and bacteria studies, the spectroscopic capability of the FTIR-SPR technique can be used to pinpoint in real time and label-free manner the structural changes in cells and bacteria. However, this imposes additional limitations, in particular, the requirement of biological compatibility practically excludes all other conducting films besides Au. In what follows we describe this FTIR-SPR technique and show several applications.

\section{Experimental Setup}

Figures 1,2 shows our experimental setup. The infrared beam is emitted from the external port of the Bruker Equinox 55 FTIR spectrometer and passes through the $1 \mathrm{~mm}$ diameter pinhole collimator. The beam diameter is $3-4 \mathrm{~mm}$ and beam divergence is $\sim 0.6^{\circ}$. The collimated beam is polarized using the grid polarizer, it is reflected from the right-angle $\mathrm{ZnS}$ prism coated with $12-20 \mathrm{~nm}$ thick $\mathrm{Au}$ film, and is focused by the parabolic mirror onto the liquid-nitrogen-cooled MCT detector. A temperature-stabilized flow chamber with analyte solution is in contact with the Au-coated base of the prism. For each incident angle we measured the reflectivity for the $s$-polarized beam and used it as a background for further measurements. Then we measured the reflectivity spectrum for the $p$-polarized beam at the same angle and identified the dip corresponding to surface plasmon resonance.

For spectroscopy of solid and liquid samples and for some living cells studies we used the vertical setup (Fig. 1) since it allows both wavelength and angular interrogation. Here the prism with attached flow chamber and the detector are mounted on the $\Theta-2 \Theta$ rotating phase. At each incident angle we analyzed the reflectivity spectra, identified the surface plasmon resonance, and determined real and imaginary part of the refractive index of the analyte solution using Fresnel reflectivity formulae.

For living cell studies, we used the horizontal setup (Fig. 2) that works at fixed incident angle and allows only wavelength interrogation. The flow chamber contains growth solution that sustains cell functioning during experiment. The prism- flow chamber assembly is mounted on the vertical translation stage. The living cells are either grown directly on prism or injected into flow chamber and allowed for sedimentation. By adding chemicals and drugs into growth solution we triggered different cell processes and studied cell response to these processes using surface plasmon resonance. The rationale for the horizontal arrangement of the flow chamber is the compatibility with the optical microscopy. Indeed, synchronously with the surface plasmon measurements, we took the optical timelapsed images of the gold-coated prism surface and the cells on it. This was done using high magnification optical zoom lens and a digital camera. 


\section{Surface Plasmon Spectroscopy}

Since wavelength $\lambda$ does not appear explicitly in Eq. 3, the resonance condition at fixed angle can, in principle, be satisfied for several wavelengths or for some range of wavelengths. This allows surface plasmon spectroscopy. To be specific, we consider the water solutions in contact with the gold-coated ZnS prism. Figure 3 shows the angle $\Theta_{\text {ext }}(\nu)$, corresponding to the surface plasmon resonance at water/Au/ZnS interface at given wave number $\nu$, using $45^{0} \mathrm{ZnS}$ prism (the angle is defined as in Fig.2). Since dielectric constant of the gold is very high, $\epsilon_{A u}>>1$, the angular range where surface plasmon can be excited is very close to the critical angle i.e., $\sin \Theta_{s p} \approx n_{\text {water }} / n_{Z n S}$. The resonant wave number can be found from the intercept of the horizontal line $\Theta=\Theta_{\text {ext }}$ with the dependence $\Theta_{\text {ext }}(\nu)$, as shown in the Figure. For $\Theta=30.5^{0}$ the surface plasmon is excited only at one wavelength, $\nu=2916 \mathrm{~cm}^{-1}$. The resonance appears in the spectral region where $\Theta \nu$ dependence is very steep, hence the width of the surface plasmon is quite modest, $\Delta \nu=200 \mathrm{~cm}^{-1}$. For $\Theta=22.5^{\circ}$ the surface plasmon is excited at several wavelengths: $\nu=1470 \mathrm{~cm}^{-1}, 1970$ $\mathrm{cm}^{-1}$ and at $5173 \mathrm{~cm}^{-1}$. The most important resonance at $\nu=5173 \mathrm{~cm}^{-1}$ appears in the spectral range where the slope of $\Theta(\nu)$ dependence is small hence the width of the resonance is extremely large $-\Delta \nu=2000 \mathrm{~cm}^{-} 1$. Both resonances are sufficiently wide to allow spectroscopy within the resonance curve even at one incident angle. Figure 4 shows corresponding experimental results. For $\Theta=30^{\circ}$ there is indeed a single narrow dip at $\nu=2916 \mathrm{~cm}^{-1}$ while for $\Theta=22^{o}$ there is a wide resonance at $5173 \mathrm{~cm}^{-1}$.

Contrary to common wisdom, the width of the surface plasmon resonance at wavelength interrogation is not directly related to losses, but is determined mostly by dispersion. The plasmon at $\nu=5173 \mathrm{~cm}^{-1}$ is very wide because the water dispersion in the corresponding spectral range is low. The losses for the narrow, $\nu=2916 \mathrm{~cm}^{-1}$, resonance are in fact higher than for the broad resonance at $\nu=5173 \mathrm{~cm}^{-1}$. The broad surface plasmon resonance allows highly sensitive spectroscopic measurements. Indeed, the weak water absorption peaks (arrows in the Fig. 4) are well resolved even without processing and deconvolution. To use the surface plasmon resonance as a spectroscopic tool, the reflectivity under the surface plasmon regime should be converted into refractive index spectra. This requires modelling of the surface plasmon reflectivity. Figure 4 shows that quite an impressive fit can be achieved using a simple optical model based on Fresnel reflectivity expressions. The fit does not take into account the interface roughness but does account for beam divergence. The fitting parameters here are the optical constants of Au film. Once these are found from the measurements at some angle, the model prediction for other angles was calculated without fitting parameters.

The next step here is to measure the surface plasmon reflectivity with an unknown analyte and to determine its refractive index spectrum using a set of spectra (similar to those in Fig.4) at different angles. We used this approach to determine the refractive index of living cell monolayer grown directly on the gold-coated $\mathrm{ZnS}$ prism. Figure 5 shows the refractive index of the cells. The refractive index of the cells generally exceeds the water refractive index since the cells contain up to $30 \%$ organic substances whose refractive index is higher than that of the water. Figure 6 shows the exploded view of the spectral region corresponding to absorption of the aliphatic bonds. For better visibility we plot there the first derivative of the refractive index. Three characteristic peaks corresponding to 


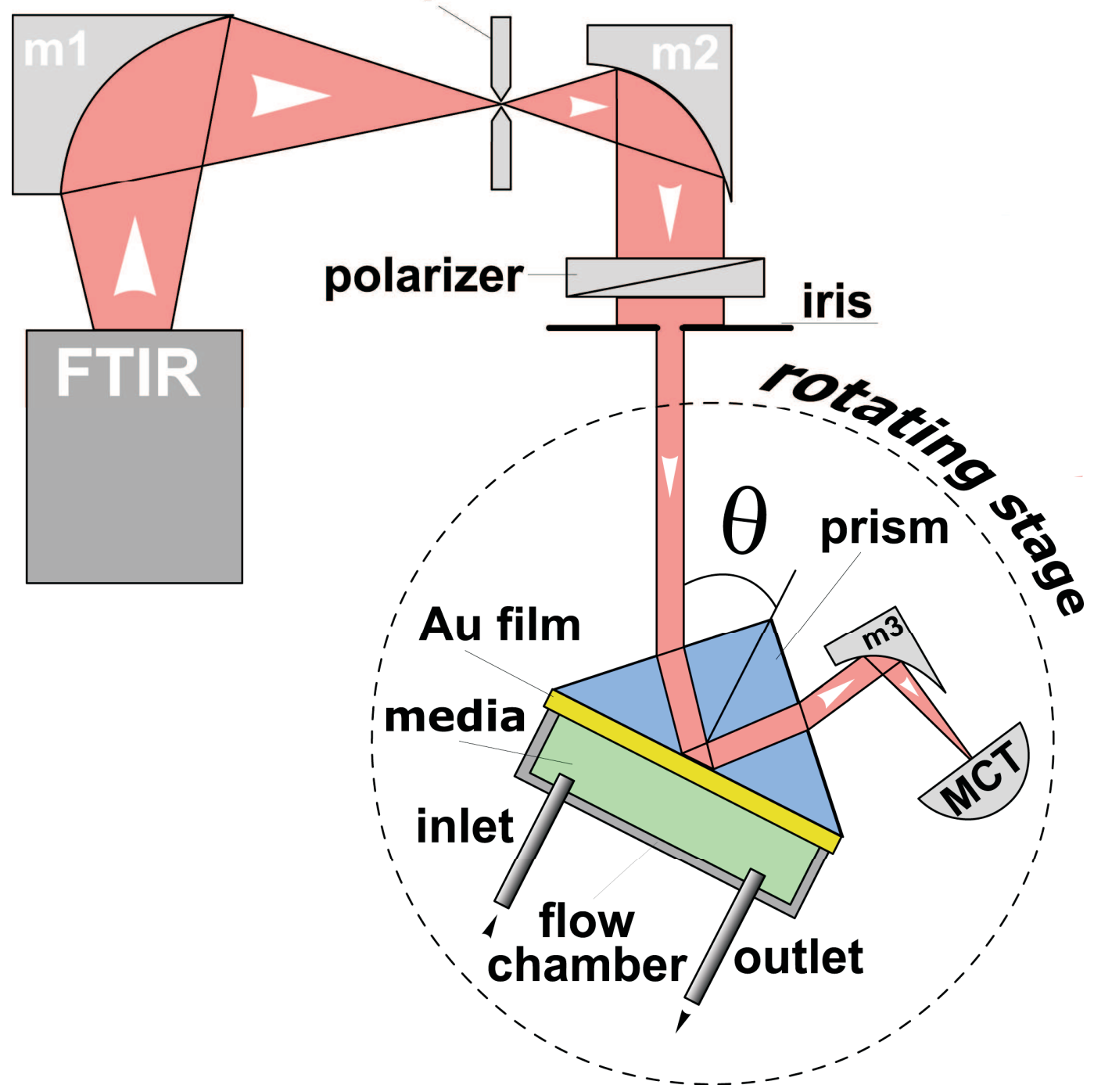

Figure 1. Surface plasmon resonance in the Kretschmann's geometry. Vertical configuration allows angular and wavelength interrogation and is useful for spectroscopy.

absorption bands of the $\mathrm{CH}_{x}$ groups are clearly visible.

\section{Living Cell Studies}

\subsection{Cell Culture}

We studied several cell lines: MDCK, HeLa, A431, human melanoma cells (MEL 1106), etc. From the point of view of the physicist, all these cells are similar in composition $(70 \%$ of water, $30 \%$ of organic substances and salts), in shape (the cell in solution is a sphere with the radius $\sim 6 \mu \mathrm{m}$ ), but they differ in their ability to spread on different substrates and 


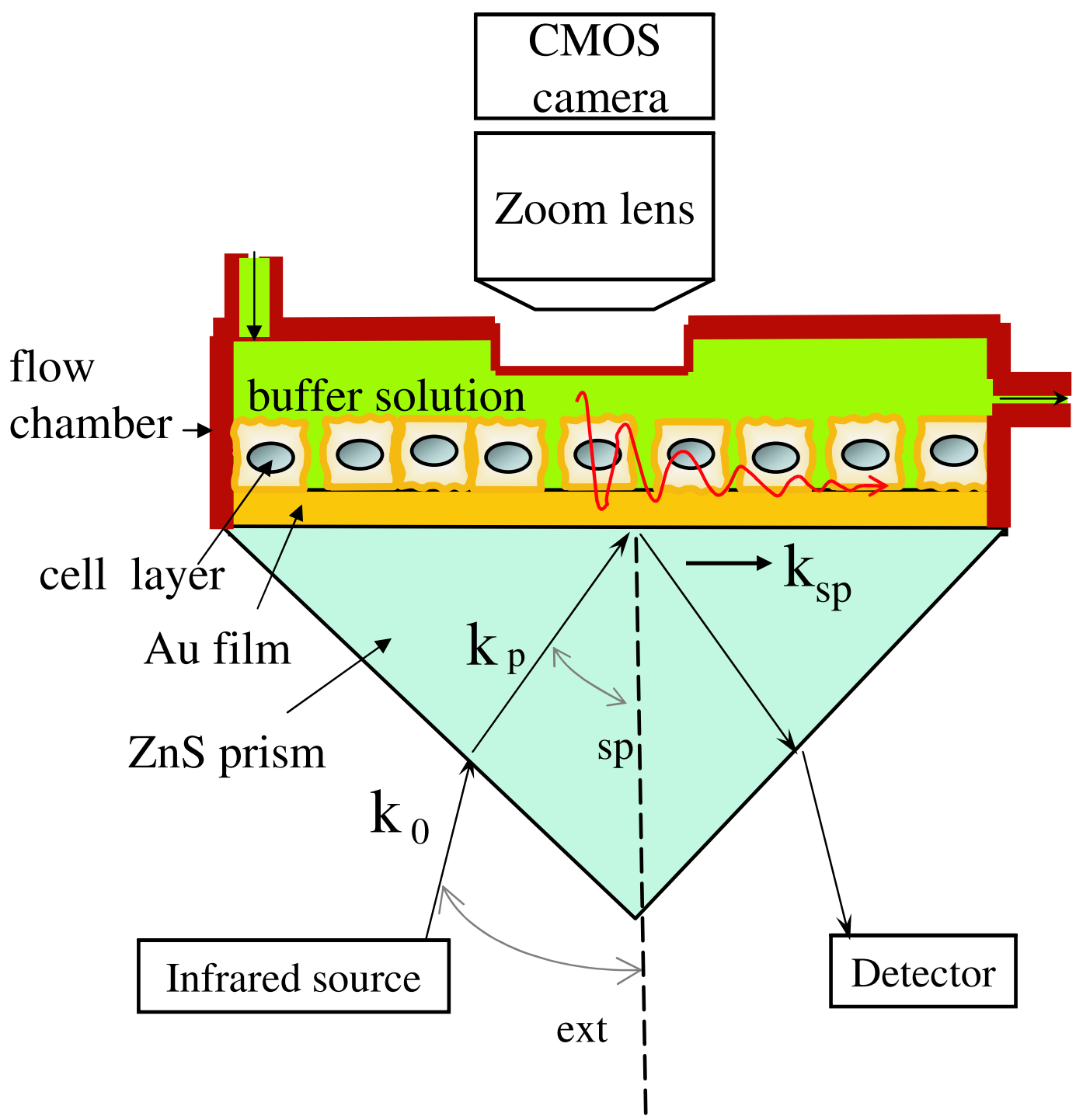

Figure 2. Surface plasmon resonance in the Kretschmann's geometry. Horizontal configuration allows wavelength interrogation and is favorable for living cell studies since it is easily combined with optical microscopy.

to form continuous monolayers. Here we focus on MDCK (Madin-Darby canine kidney) cells. These are epithelial cells that have a strong tendency to form continuous monolayer. In this work we study the MDCK cell sedimentation and spreading on substrate.

The MDCK cells were cultured routinely in modified Eagle's medium (MEM, Biological Industries, Israel), supplemented with $4.5 \mathrm{~g} / \mathrm{l}$ D-glucose, $10 \%$ antibiotics, and $10 \%$ fetal calf serum, as described in Ref. (4). A confluent MDCK monolayer cultured on a $10-\mathrm{cm}$ dish, was washed twice with pre-warmed PBS and the cells were detached from the dish with trypsin/EDTA (0.25 \% Trypsin/EDTA in Puck's saline A; Biological Industries, Israel). Following trypsinization, the cells were resuspended in $10 \mathrm{ml}$ minimum essential 


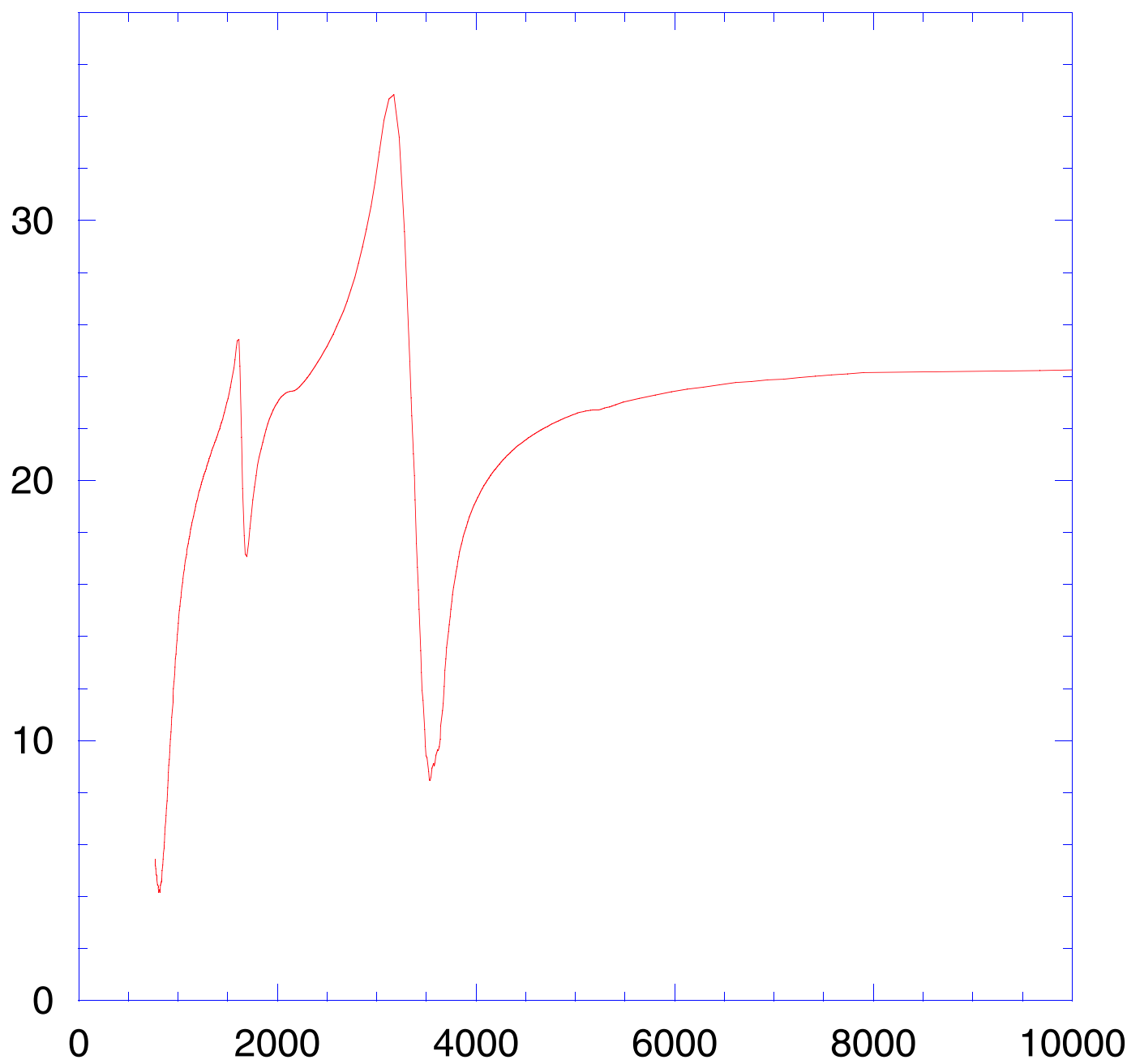

Figure 3. Incident angle corresponding to the excitation of the surface plasmon resonance at the water/ $\mathrm{Au} / \mathrm{ZnS}$ interface using $45^{0} \mathrm{ZnS}$ prism. For $\Theta=30.5^{0}$, the surface plasmon is excited at a single wave number, $2916 \mathrm{~cm}^{-1}$, and its with is only $200 \mathrm{~cm}^{-1}$. For $\Theta=22.5^{0}$, the surface plasmon is excited at $5173 \mathrm{~cm}^{-1}$ and its width is $2000 \mathrm{~cm}^{-1}$ The angle, $\Theta_{\text {ext }}$, is defined as in Fig.2.

medium (MEM) Hanks' salts supplemented with 20mM Hepes, (pH7.5) and 10\% fetal calf serum.

\subsection{Experimental Protocol}

(a) The flow chamber was attached to the prism and filled with growth medium. The prism-flow chamber assembly was incubated for 2 hours at $37^{0} \mathrm{C}$. Figure 7 a shows the optical micrograph of the Au-coated prism exposed to growth medium (prior to cell sedimentation).

(b) The growth medium with cell suspension $\left(\sim 5 \times 10^{5}\right.$ cells $\left./ \mathrm{ml}\right)$ was injected at a rate 


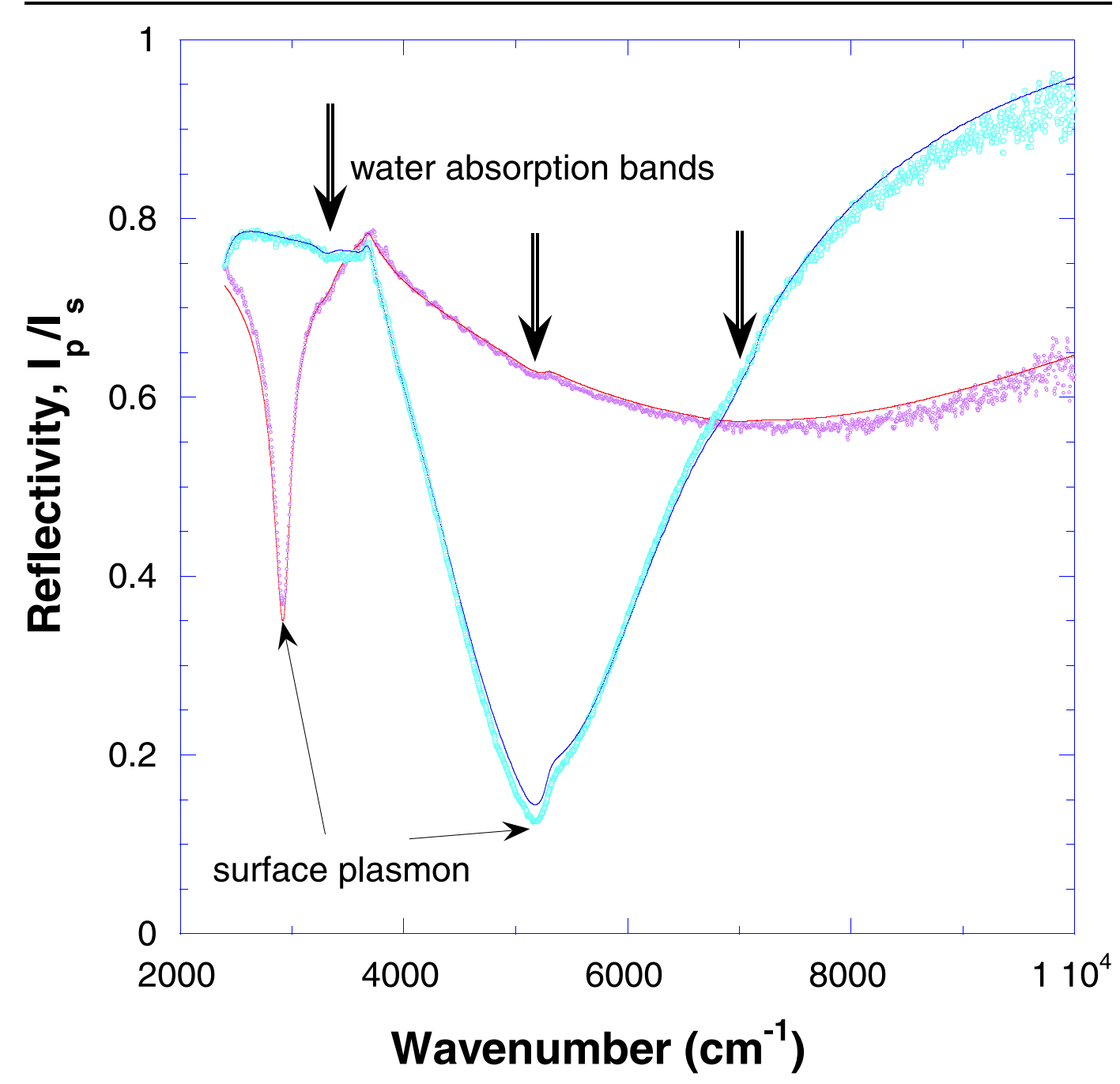

Figure 4. Infrared reflectivity from the water/Au/ZnS interface under Kretschmann's configuration at two incident angles. The pink curve corresponds to a narrow-band surface plasmon excited in the spectral range where water dispersion is strong. The blue curve corresponds to the surface plasmon excited in the spectral range where the the water dispersion is weak. Broad surface plasmon explicitly reveals absorption peaks of water (double arrows). Continuous lines indicate model prediction using known optical constants of water. Note excellent agreement to experimental data.

of $200 \mu \mathrm{l} / \mathrm{min}$. The injection continued for 15 minutes and another 5 minutes were allowed for cells in solution to sediment onto the substrate. Figure $7 \mathrm{~b}$ shows that at the end of the sedimentation phase the gold substrate is covered by spherical cells that do not contact each other. The surface cell density at the end of the injection phase was $C_{s} \sim 1700$ cells $/ \mathrm{mm}^{2}$.

(c) The growth medium without cells was injected under constant flow rate of $25 \mu \mathrm{l} / \mathrm{min}$ during $\sim 400$ minutes. During this time the cells spread on the substrate, lose their 
spherical shape and become more flat in such a way that the cell projection grows (Fig. 7c). Then the cell-cell attachment occurs and the clusters of several cells are formed. Eventually the clusters merge and develop a loose percolating cluster. Finally, the cells form a continuous monolayer with few isolated voids that are slowly closed over the time. The cell surface density almost didn't increase during the measurements since the duration of our experiment was too short in order that considerable cell proliferation occur.

(d) The growth medium flow was stopped and $0.05 \%$ Trypsin/EDTA solution was introduced. This results in disruption of the cell-substrate and cell-cell contacts. Figure $7 \mathrm{~d}$ shows that the cells regained their spherical shape while they are still residing on the substrate.

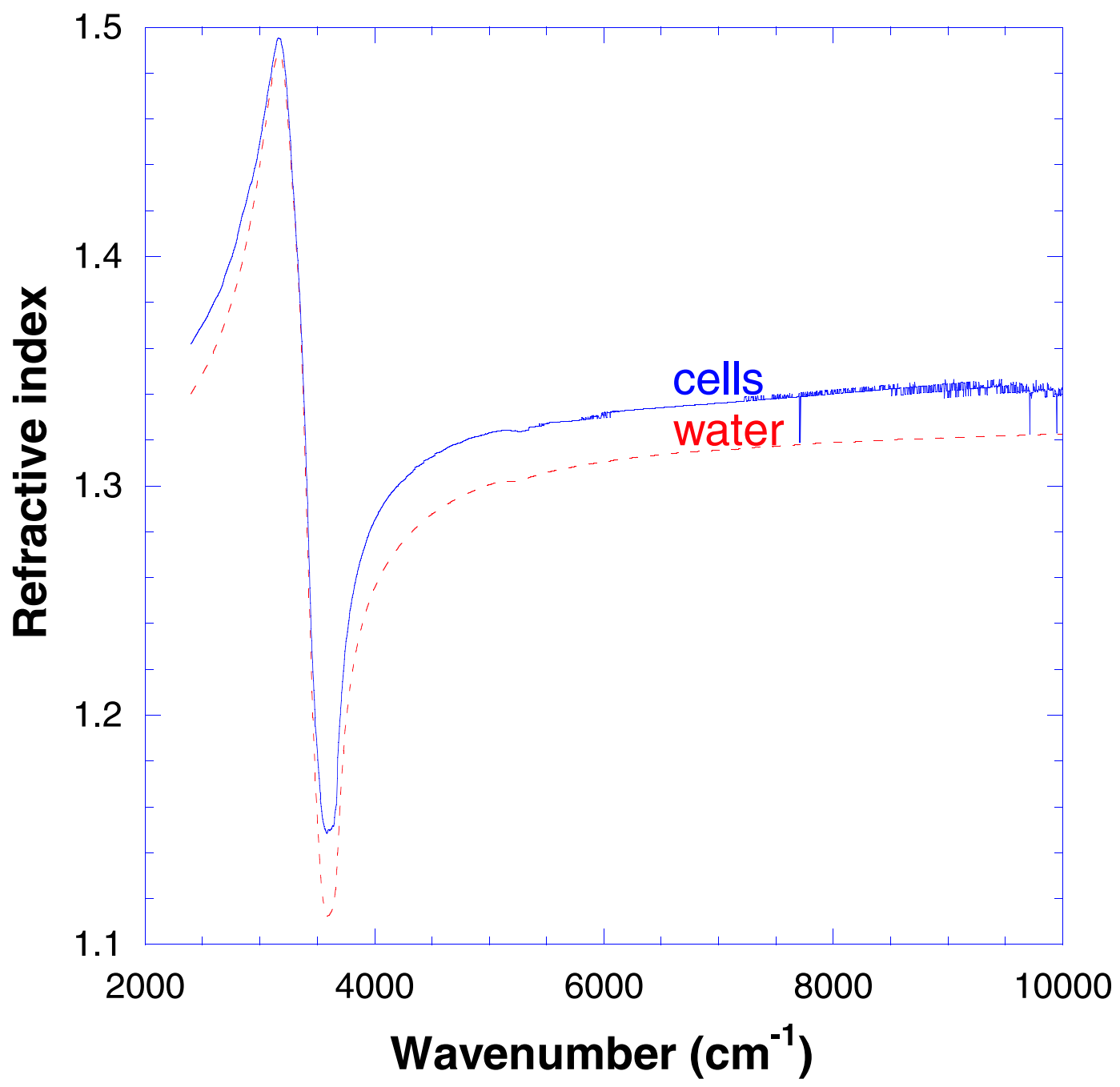

Figure 5. Refractive index of the living MDCK cell monolayer. 


\subsection{SPR Measurements of Cell Sedimentation and Spreading on Substrate}

\subsubsection{SPR spectra}

All these processes were monitored by the FTIR-SPR technique. Each FTIR spectrum taked $25 \mathrm{sec}$. (averaging over 8 scans, $8 \mathrm{~cm}^{-1}$ resolution, incident angle $\Theta_{\text {ext }}=21.35^{0}$ ) and was synchronized with the optical time-lapsed imaging of the flow chamber contents.

Figure 8 shows reflectivity spectra from the growth medium/cells/Au/ZnS prism under different phases of cell sedimentation and spreading. The black open symbols show reflectivity from the gold-coated $\mathrm{ZnS}$ prism in contact with growth medium, before cell seeding (see Fig.7a). The surface plasmon resonance at $4263 \mathrm{~cm}^{-1}$ is clearly seen. The feature at $5180-5310 \mathrm{~cm}^{-1}$ arises from the water absorption peak.

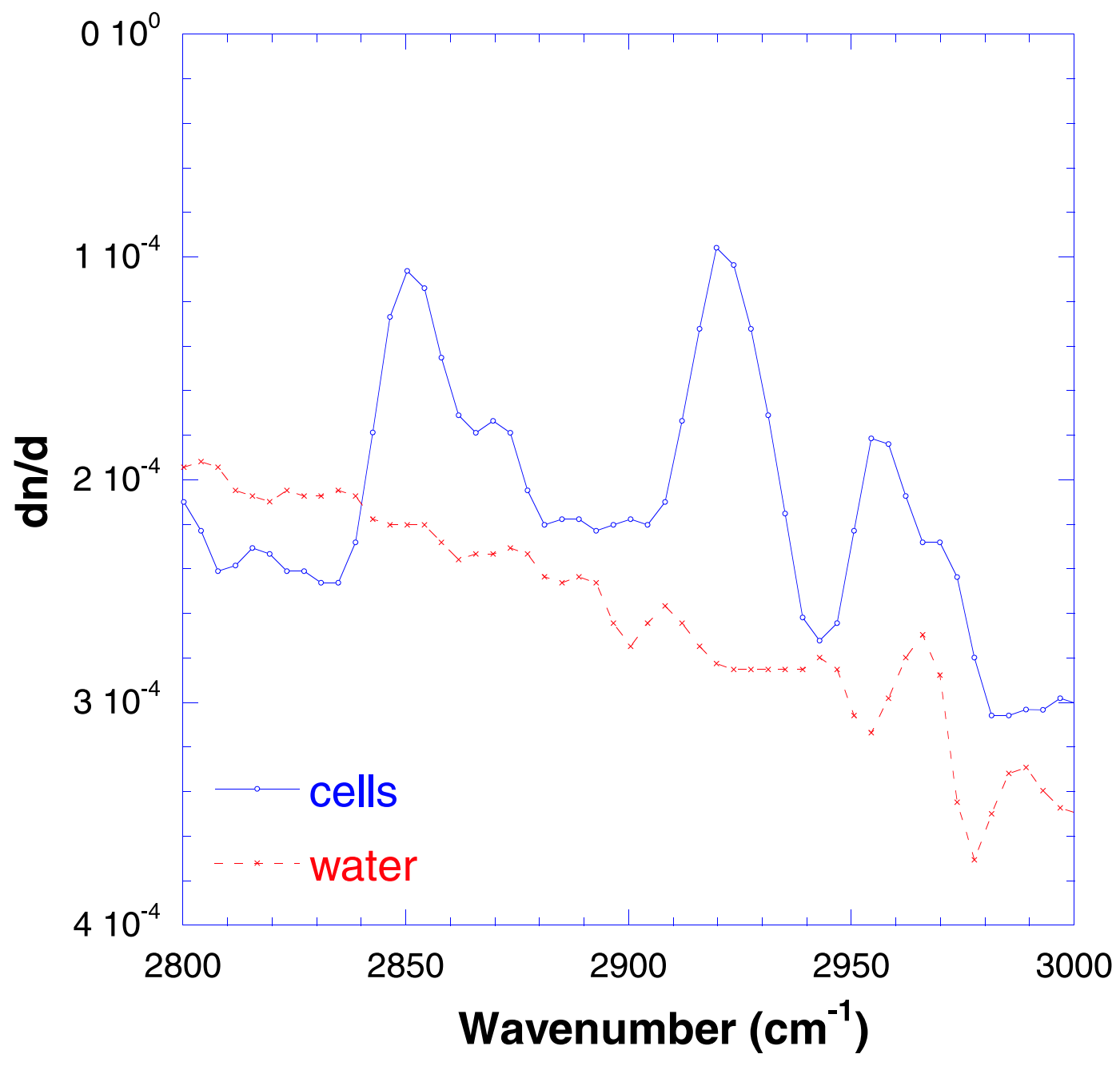

Figure 6. First derivative of the refractive index of the living MDCK cell monolayer. Note three peaks corresponding to $\mathrm{CH}_{x}$ absorption bands. The red dashed curve shows corresponding data for pure water calculated using the data of Ref.[35].

Upon formation of cell monolayer (Fig.7c), the surface plasmon resonance shifts from 


\section{Bare $\mathrm{Au} \quad$ Deposition Spreading Detachment}

(a)

(b)

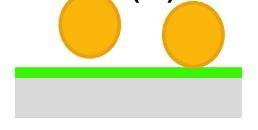

(c)

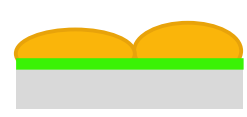

(d)

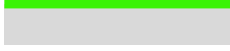

Figure 7. Optical micrographs of the Au-coated ZnS prism and the MDCK cells thereon at different phases of sedimentation, spreading, and trypsinization. The schematic drawing indicates cell shape during these phases.

$4263 \mathrm{~cm}^{-1}$ to $3903 \mathrm{~cm}^{-1}$. This $390 \mathrm{~cm}^{-1}$ shift corresponds to increased refractive index of the cells with respect to that of the growth medium (mostly water), as it is shown in Fig.5. The dip at $4470 \mathrm{~cm}^{-1}$ arises from the guided mode resonance in the cell monolayer. This dip actually signifies formation of continuous cell monolayer, as expected for epithelial cells.

Following trypsinization, the cell-cell and cell-substrate contacts are disrupted and the cells regain their spherical shape, while touching the substrate only at the apex of the sphere (Fig.7d). Then, the surface plasmon propagates mostly along the growth medium-Au interface and almost does not feel the cells. In accordance with this scenario, the surface plasmon resonance has been shifted back to $4250 \mathrm{~cm}^{-1}$ and the guided mode resonance disappeared. The degree of the surface plasmon penetration into cells can be judged from the very small residual $13 \mathrm{~cm}^{-1}$ shift of the surface plasmon resonance (see inset). The presence of cells on substrate is also manifested by the strong decrease of reflectivity at $4400-4900 \mathrm{~cm}^{-1}$. We believe that this feature is strongly sensitive to scattering on cells.

\subsubsection{Which cell properties are measured by the surface plasmon resonance tech- nique?}

We demonstrate here that the surface plasmon resonance senses the surface cell concentration $C_{s}$ and cell projection area, $A$. For quantitative determination of these parameters we generally followed the model developed by Refs. [29,30]. This model assumes that the cell initially attaching to the substrate has a spherical shape with radius $r_{0}$. Upon cell spreading, its shape changes from the sphere with the volume $V=4 \pi r_{0}^{3} / 3$ to the spherical cap (see Fig.7c) with the volume, $V=\frac{\pi h}{6}\left(h^{2}+3 r^{2}\right)$. Here, $h$ is the cell height over the substrate and $r$ is the curvature radius of the cell surface. Since the cell volume is generally preserved upon spreading, this results in some inexplicit relation between $r$ and $h$.

The surface plasmon shift upon cell sedimentation is determined by the effective refrac- 
tive index of the cells immersed in growth medium [29]:

$$
\Delta n=C_{s} \int_{0}^{\infty}\left(n_{\text {cell }}-n_{\text {medium }}\right) A(z) e^{-\frac{z}{\delta}} \frac{d z}{\delta}
$$

where $C_{s}$ is the cell concentration, $n_{\text {cell }}$ and $n_{\text {medium }}$ are refractive indices of the cell and of the growth medium, correspondingly, $\delta$ is the surface plasmon penetration depth, and $A(z)$ is the cross-section of the cell at the height $z$ above the substrate.

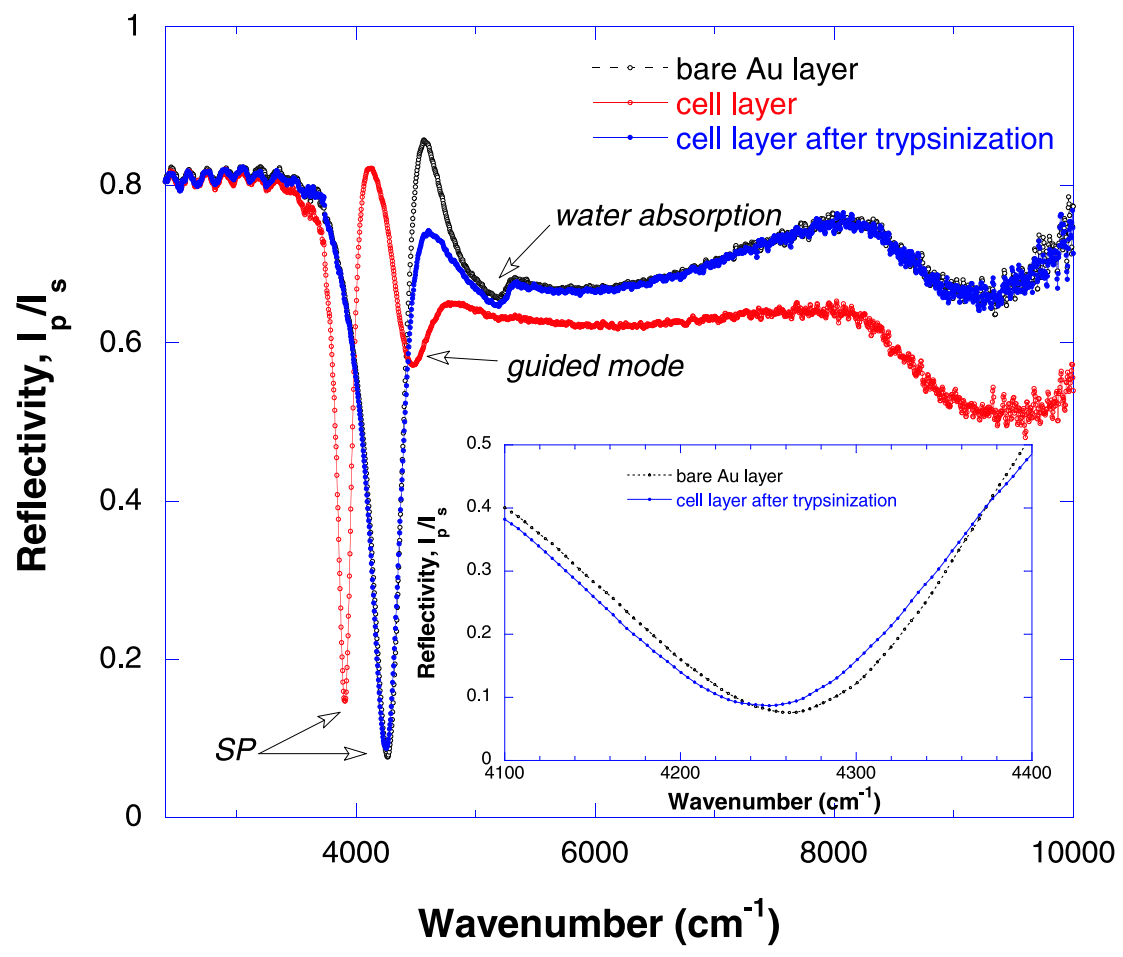

Figure 8. Infrared reflectivity spectra from the layer of MDCK cells on Au-coated $\mathrm{ZnS}$ prism. The open black circles indicate reflectivity from the bare Au-coated $\mathrm{ZnS}$ prism exposed to growth solution consisting mostly of water. (This corresponds to Fig.7a). Note the surface plasmon dip at $4263 \mathrm{~cm}^{-1}$. The feature at $5173 \mathrm{~cm}^{-1}$ corresponds to the water absorption peak. The red filled circles indicate reflectivity from the prism with a confluent MDCK cell monolayer (This corresponds to Fig.7c). The surface plasmon dip has been shifted to $3900 \mathrm{~cm}^{-1}$ and the guided mode dip at $4470 \mathrm{~cm}^{-1}$ has appeared. The filled blue circles indicate reflectivity from the cell layer on the prism immediately after adding a small quantity of trypsin (This corresponds to Fig.7d). The trypsinization disrupts the cellcell and cell-substrate contacts, the cells regain the spherical shape and their contact with the substrate is minimized. Note, that the SP dip has been shifted back to $4250 \mathrm{~cm}^{-1}$ and the guided mode peak disappeared. The reflectivity at $4400-4900 \mathrm{~cm}^{-1}$ has been decreased indicating increased SP scattering. The inset zooms into the SP dip. The small difference between the reflectivity of bare Au substrate and the substrate with spherical cells is visible, indicating that the cells were not washed out.

Equation 9 suggests that during the sedimentation phase, the surface plasmon measures 
cell concentration, $C_{s}$. After sedimentation phase is over, the surface plasmon resonance shift measures the cell cross-section weighted over surface plasmon penetration depth $(\delta \approx$ $2 \mu \mathrm{m}$ for our wavelength range). At the end of the spreading process the cells form monolayer with the height $h \sim 8 \mu \mathrm{m}$. Since $h>\delta$, this means that at least at the advanced phases of cell spreading, Eq.9 reduces to $\Delta n \approx C_{s}\left(n_{\text {cell }}-n_{\text {medium }}\right) A$ where $A=\pi h(2 r-h)$ is the cell projection area i.e., the cell cross-section as seen by the optical microscopy. This was verified by our simultaneous optical microscopy and surface plasmon measurements.

The reflectivity under the surface plasmon resonance is determined mostly by scattering, since our range of wavelengths does not include the absorption bands of cell constituents. The scattering of surface plasmon waves has been extensively studied [33] mostly for regime of small and strong scatterers. The cell size considerably exceeds the wavelength and it is the weak scatterer, since $n_{\text {cell }} / n_{\text {medium }} \sim 1$. In the absence of appropriate theoretical model describing surface plasmon scattering on such large and weak scatterers as cells, we restrict ourselves to qualitative treatment of the problem based on the Beer's law for surface waves. This law states that the imaginary part of the wave vector is proportional to the concentration of scatterers, $k_{s p}^{\prime \prime}=C_{s} l_{\text {cell }}$, where $l_{\text {cell }}$ is the scattering cross-section of a single cell. Since the cells are big scatterers whose size exceeds the surface plasmon wavelength by an order of magnitude, it is natural to assume that the scattering occurs at cell-growth medium interface. Then $l_{\text {cell }} \propto P$ where $P$ is the cell perimeter. This reasoning reduces to conjecture that the surface plasmon reflectivity measures the length of the cell-growth medium interfaces per unit area.

\subsubsection{Kinetics of cell spreading}

Time dependence of the surface plasmon shift $\Delta \nu$ and reflectivity $R_{\text {min }}$, yields kinetics of cell sedimentation and spreading. Figure 9 shows how the surface plasmon resonance has been shifted with time. The SPR shift varies fast during cell spreading phase and goes to saturation after $50 \mathrm{~min}$. At this phase $\Delta \nu \propto C_{s}$ since the cells are still spheres and $A$ is constant. After cell sedimentation is over, $\Delta \nu$ grows slowly and this growth is associated with cell spreading i.e., increase of $A$. This $\Delta \nu$ growth is not steady but goes through several phases, representing different strategies of cell spreading.

These phases are also seen in the reflectivity. $R_{\min }$ grows fast upon cell sedimentation due to increasing cell concentration $C_{s}$ and goes to saturation after sedimentation is over. However, after $80 \mathrm{~min}$. the reflectivity starts to grow again, passes through the maximum, and decreases. After trypsinization, the reflectivity and the shift vary in reverse order (not shown here) and almost regain their initial values before cell spreading.

Basing on our simultaneous optical measurements we validated the above scenario, determined the cell projection and found surface coverage, $f=C_{s} A$. Following the approach adopted in the context of thin film growth, we plot the data of the Fig.10 as reflectivity (in other words, interface perimeter per unit area) versus surface coverage. Figure 10 shows different phases of cell spreading. The end of the cell sedimentation phase corresponds to $f=0.025$. After sedimentation is over, the surface coverage grows due to spreading of individual cells. When $f=0.5-0.6$ the cells already contact one another and their further spreading is impeded. This bears some resemblance to formation of percolating cluster.

When $f \approx 0.6$, the cells obviously switch to another spreading regime, as revealed by 


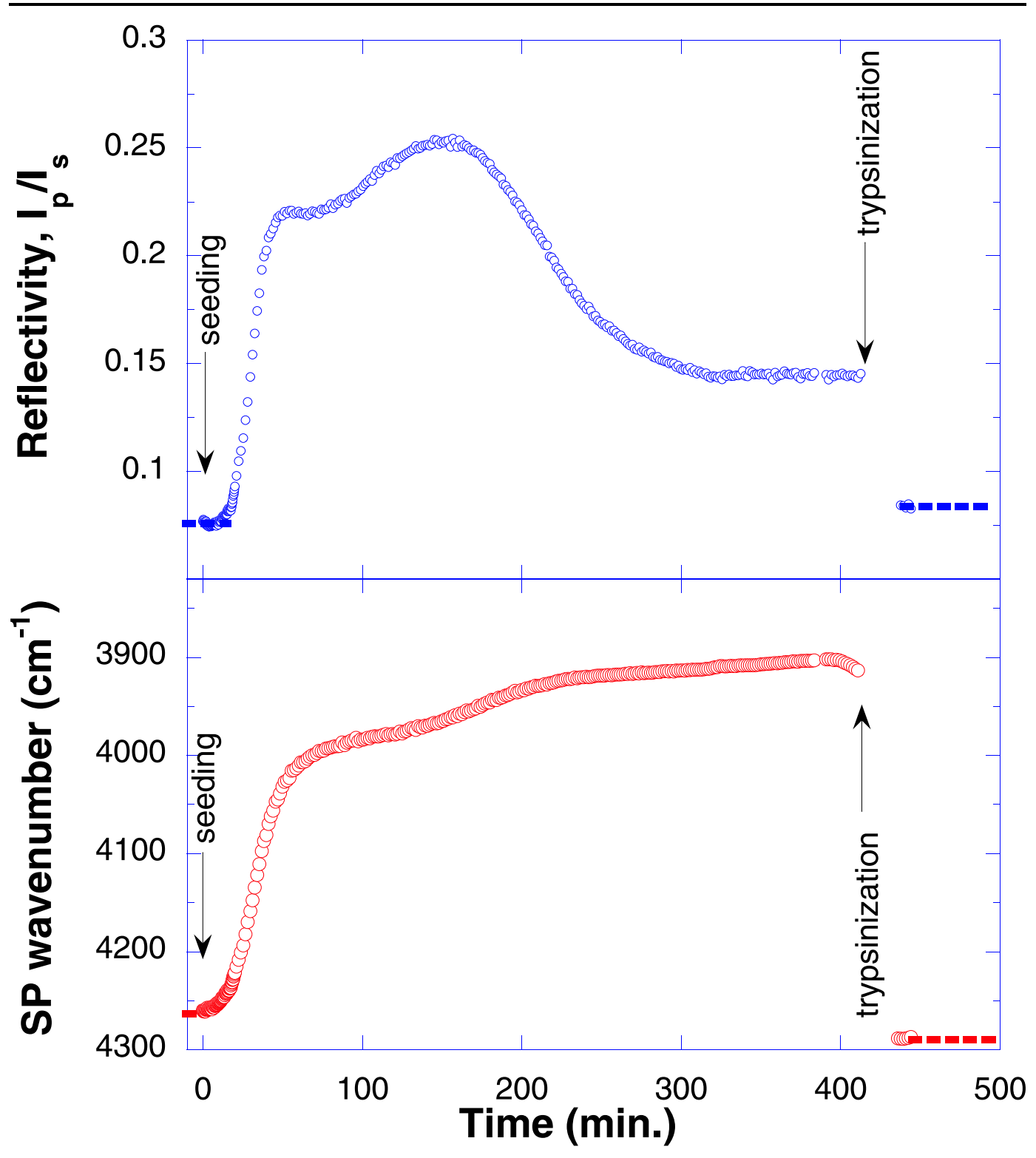

Figure 9. Time dependence of the surface plasmon shift $\nu_{\min }$ (lower panel) and the reflectivity at the surface plasmon dip, $R_{\min }$ (upper panel) during cell sedimentation and spreading. $t=0$ corresponds to cell injection. Upon cell sedimentation and spreading, the cell mass which is in close contact with prism increases and the SP resonant wave number is constantly shifted to the red, indicating increasing refractive index sensed by the SP wave. The $R_{\min }$ is mostly determined by the scattering on cells and varies non monotonously through the cell deposition process. After trypsinization, when the cells regain the spherical shape and are eventually washed out, the SP wavenumber and reflectivity almost reversibly return to their initial values before cell deposition.

increase in reflectivity without marked increase in the surface plasmon shift. We believe 


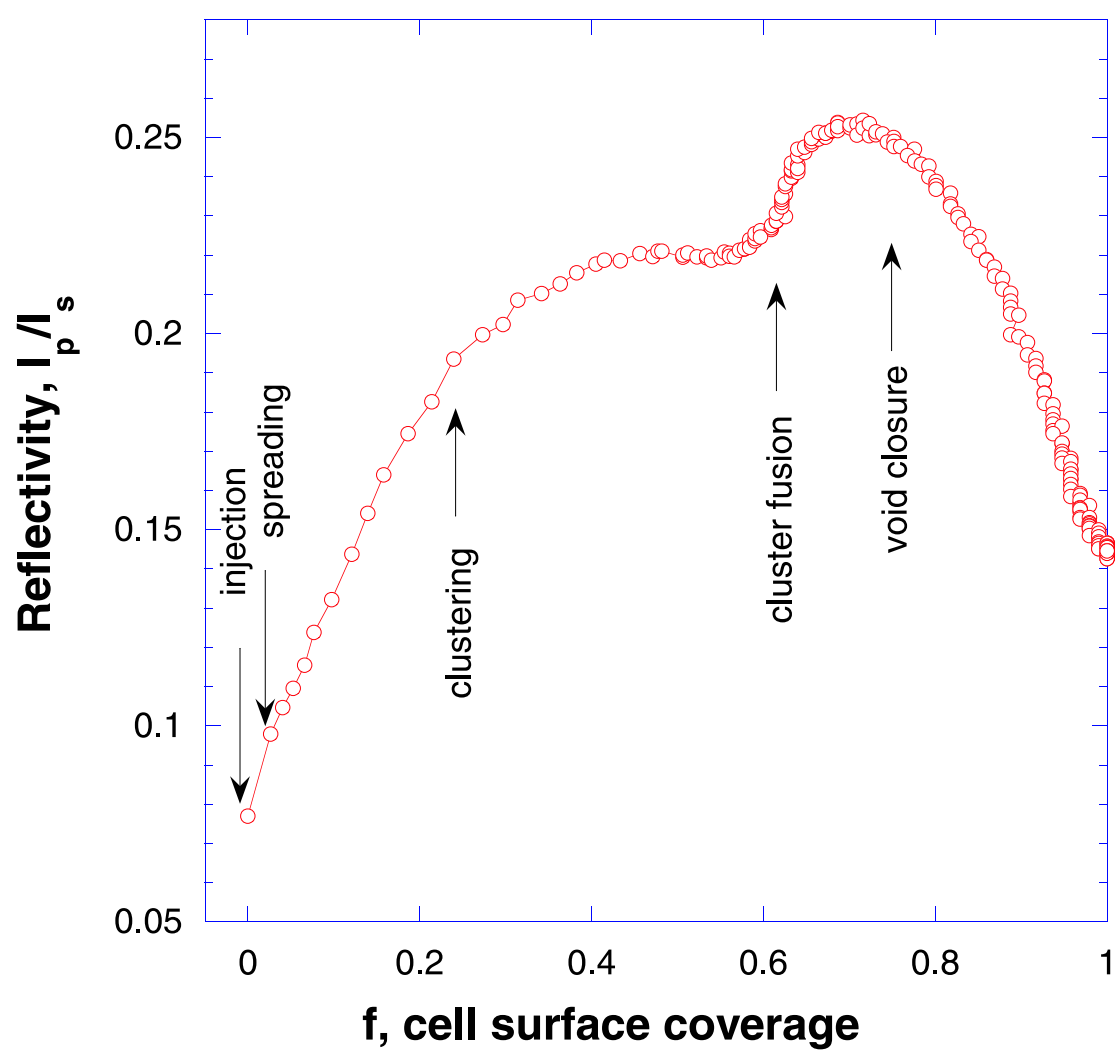

Figure 10. Reflectivity at the dip of the surface plasmon resonance, $R_{\min }$, versus cell surface coverage, $f$, during cell spreading. The range below $f=0.6$ indicates individual cell spreading followed by clusters formation. The range above $f=0.6$ is probably related to another spreading regime where the cells seek the voids in the cell layer and try to fill them. This is associated with enhanced surface plasmon scattering.

that for $f<0.6$ the cells spread and occupy the nearby areas of substrate. When $f<0.6$ the cell layer becomes too crowded and the cells should explore the remote places as well. It is known that to spread the cells send thin lamellipodia [36] to explore the substrate and to find unoccupied areas. Using this knowledge the cells cover these areas in such a way that the continuous monolayer is formed. We believe that this spreading regime is associated with increased lamellipodia production. While the lamellipodia perimeter is large (hence they are seen through reflectivity), their thickness $(0.1-0.3 \mu \mathrm{m})$ is significantly smaller than the surface plasmon penetration depth, hence the surface plasmon shift is less affected.

The reflectivity sharply drops when the filling factor exceeds $f=0.75$. Incidentally, this threshold is very close to that of random close packing of hard disks, $f_{R C P}=0.82$ [34]. The reason for the reflectivity drop is the decrease of scattering associated with void shrinking in cell monolayer. 


\section{Conclusions}

We demonstrated here that the infrared surface plasmon can be used as a spectroscopic tool to measure infrared spectra in a thin layer. There are two distinctive features that make surface plasmon so different from conventional infrared spectroscopy techniques, such as attenuated total reflection and optical fibers. First, the surface plasmon measures refractive index in such a way that it is a complementary method to ATR that measures absorption. We developed an optical model of the reflectivity from multilayers in the regime of the surface plasmon resonance. This model allows quantitative determination of the refractive index spectrum of the analyte. Second, the surface plasmon has larger penetration depth ( $\sim 1 \mu \mathrm{m}$ as compared to $0.5-0.7 \mu \mathrm{m}$ in ATR). This is especially advantageous for living cell studies since surface plasmon probes the cell interior. The potential of the surface plasmon resonance for quantitative real-time and label-free studies of living cells on substrate has been demonstrated.

\section{Acknowledgments}

This research has been supported by the Israeli Ministry of Industry and Trade through the NOFAR program.

\section{References}

[1] H. Raether, Springer Tracts in Modern Physics 111, 1 (1988).

[2] S.A. Maier, Plasmonics: applications and fundamentals, 2007, Springer Science+Business Media LLC.

[3] W. Knoll, Annu. Rev. Phys. Chem. 49, 569 (1998).

[4] J. Homola, Chem. Rev. 108, 462 (2008).

[5] A. G. Frutos, S. C. Weibel, and R. M. Corn, Anal. Chem. 71, 3935 (1999).

[6] B. P. Nelson, A. G. Frutos, J. M. Brockman, and R. M. Corn, Anal. Chem. 71, 3928 (1999).

[7] A. Ikehata, T. Roh, and Y. Ozaki, Anal. Chem. 76, 6461 (2004).

[8] S. Patskovsky, A. V. Kabashin, M. Meunier, J. H.T. Luong, Sensors and Actuators B 97, 409 (2004).

[9] J.F. Masson, Y.C. Kim, A. Loius, L.A. Obando, W. Peng, and K. S. Booksch, Appl. Spectroscopy 60, 1241 (2006).

[10] V. Lirtsman, M. Golosovsky, D.Davidov, J. Appl. Phys. 103, 014702 (2008).

[11] M. Golosovsky, Lirtsman, V., Yashunsky, V., Davidov, D., and Aroeti, B. Journal of Applied Physics 105, 102036 (2009) 
[12] R. Ziblat, V. Lirtsman, D. Davidov, and B. Aroeti, Biophys. J. 91, 776 (2006).

[13] V. Yashunsky, S. Shimron, V. Lirtsman, A.M. Weiss, N. Melamed-Book, M. Golosovsky, D. Davidov, B. Aroeti, Biophys. J. 97, 1003 (2009).

[14] K.F. Giebel, C. Bechinger, S. Herminghaus, M. Riedel, P. Leiderer, U. Weiland, and M. Bastmeyer, Biophys. J. 76, 509 (1999).

[15] Y. Fang, A. M. Ferrie, N. H. Fontaine, J. Mauro, and J. Balakrishnan, Biophys. J. 91, 1925 (2006).

[16] Y. Yanase, H. Suzuki, T. Tsutsui, I.Uechi, T. Hiragun, S. Mihara, M. Hide, Biosensors and Bioelectronics 22, 1081 (2007).

[17] Cuerrier, V.C. Chabot, S. Vigneux, V. Aimez, E. Escher, F. Gobeil Jr, P.G. Charette, M. Grandbois, Cellular and Molecular Bioengineering, 1, 229 (2008).

[18] A.A. Kolomenskii, P. D. Gershon, and H. A. Schuessler, Appl. Optics 39, 3314 (2000).

[19] M. Zangeneh, N.Doan, E.Sambriski, and R. H. Terrill, Appl. Spectroscopy 58, 10 (2004).

[20] J.V. Coe, K.R. Rodriguez, S. Teeters-Kennedy, K. Cilwa, J. Heer, H. Tian, S.M. Williams, J. Phys. Chem. 111, 17459 (2007).

[21] L.M. Miller, P. Dumas, Biochimica et Biophysica Acta 1758, 846 (2006).

[22] M.K. Kuimova, K.L.A. Chan, and S.G.Kazarian, Appl. Spectroscopy 63, 164. (2009)

[23] U. Zelig, J.Kapelushnik, R. Moreh, S.Mordechai, and I. Nathan, Biophys. J. 97, 2107 (2009).

[24] Hastings, G., Wang, R., Krug, P., Katz, D., and Hilliard, J. Biopolymers 89, 921 (2008)

[25] A. Hirano-Iwata, R. Yamaguchi, K.Miyamoto, Y.Kimura, M. Niwano, J. Appl. Phys. 105, 102039 (2009).

[26] H.Y. N.Holman, M.C. Martin and W.R. McKinney, Spectroscopy 17139 (2003).

[27] Y.Raichlin, A.Katzir, Appl. Spectroscopy 62, 55a (2008).

[28] K. Johansen, H. Arwin, I. Lundstrom, and B. Liedberg, Rev. Sci. Instr. 71, 3530 (2000).

[29] J.J.Ramsden, S.Y. Li, E. Heinzle, and J. E. Prenosil, Cytometry 19, 97 (1995).

[30] J.J. Ramsden, and R. Horvath, J. Receptors and Signal Transduction, 29, 211 (2009).

[31] R. Horvath, K. Cottier, H.C. Pedersen, and J.J. Ramsden, Biosensors and Bioelectronics, 24, 799 (2008). 
[32] T.S. Hug, J. E. Prenosil, and M. Morbidelli Biosensors and Bioelectronics 16, 865 (2001).

[33] T.A.Leskova, A.A. Maradudin, I.V. Novikov, J. Opt. Soc. Am. 17a, 1288 (2000).

[34] D. Bideau, A.Gervois, L. Oger, J.P. Troadec, J. de Physique 47, 1697 (1986).

[35] J.E. Bertie, Z. Lan, Appl. Spectroscopy 50, 1047 (1996).

[36] P.K. Mattila, P. Lappalainen, Nature reviews, Molecular cell biology 9, 446 (2008). 